\title{
BERNSTEIN OPERATORS FOR EXTENDED CHEBYSHEV SYSTEMS
}

\author{
J.M. ALDAZ, O. KOUNCHEV AND H. RENDER
}

\begin{abstract}
Let $U_{n} \subset C^{n}[a, b]$ be an extended Chebyshev space of dimension $n+1$. Suppose that $f_{0} \in U_{n}$ is strictly positive and $f_{1} \in U_{n}$ has the property that $f_{1} / f_{0}$ is strictly increasing. We search for conditions ensuring the existence of points $t_{0}, \ldots, t_{n} \in$ $[a, b]$ and positive coefficients $\alpha_{0}, \ldots, \alpha_{n}$ such that for all $f \in C[a, b]$, the operator $B_{n}$ : $C[a, b] \rightarrow U_{n}$ defined by $B_{n} f=\sum_{k=0}^{n} f\left(t_{k}\right) \alpha_{k} p_{n, k}$ satisfies $B_{n} f_{0}=f_{0}$ and $B_{n} f_{1}=f_{1}$. Here it is assumed that $p_{n, k}, k=0, \ldots, n$, is a Bernstein basis, defined by the property that each $p_{n, k}$ has a zero of order $k$ at $a$ and a zero of order $n-k$ at $b$.

2000 Mathematics Subject Classification: Primary: 41A35, Secondary 41 A50

Key words and phrases: Bernstein polynomial, Bernstein operator, extended Chebyshev system, exponential polynomial
\end{abstract}

\section{INTRODUCTION}

A recent development in CAGD is the analysis of properties of Bernstein bases defined by trigonometric or hyperbolic polynomials, or more generally, by elements in a Chebyshev space, see [4], [5], [6], [7], [13], [14], [15], [16], [17], [19], [20], [21]. The definition of a Bernstein basis is easily stated: Let $K$ be either the field of real or complex numbers, denoted by $\mathbb{R}$ and $\mathbb{C}$ respectively. Assume that $U_{n}$ is a $K$-linear subspace of dimension $n+1$ of $C^{n}(I, K)$, the space of $n$-times continuously differentiable $K$-valued functions on an interval $I=[a, b]$. A system $p_{n, k}, k=0, \ldots, n$, in $U_{n}$ is called a Bernstein basis for $\{a, b\}$ if the function $p_{n, k}$ has a zero of order $k$ at $a$ and a zero of order $n-k$ at $b$, for $k=0, \ldots, n$. Unlike the case of the classical Bernstein polynomial basis on $[a, b]$, the preceding definition does not exclude the possibility that $p_{n, k}$ might have additional zeros inside the open interval $(a, b)$. It is easy to see that a Bernstein basis is indeed a basis of the linear space $U_{n}$ and that the basis functions are unique up to a non-zero factor, see e.g. the proof of Lemma 19 and Proposition 20 in [11. The existence of a Bernstein basis is related to the concept of an extended Chebyshev system: A $K$-linear subspace $U_{n} \subset C^{n}(I, K)$ of dimension $n+1$ is an extended Chebyshev system (or space) for the subset $A \subset I$ if each non-zero $f \in U_{n}$ vanishes at most $n$ times in $A$, counting multiplicities. No condition is imposed regarding the zeros of $f$ in $I \backslash A$. It is not difficult to prove that a Bernstein basis exists for $U_{n} \subset C^{n}(I, K)$ if and only if $U_{n}$ is an extended

The first and the last author are partially supported by Grant MTM2006-13000-C03-03 of the D.G.I. of Spain. The last two authors acknowledge support within the project "Institutes Partnership" with the Alexander von Humboldt Foundation, Bonn. 
Chebyshev system for the set $\{a, b\}$, see e.g. [4], [15] for the case $K=\mathbb{R}$. In the complex case $K=\mathbb{C}$, Corollary 21 in [11] gives the necessity; a simple argument from linear algebra gives the existence of a function $f_{k} \in U_{n}, f_{k} \neq 0$, having at least $k$ zeros at $a$ and at least $n-k$ zeros $b$, and using that $U_{n}$ is a Chebyshev system for $\{a, b\}$ one concludes that $f_{k}, k=0, \ldots, n$, is indeed a Bernstein basis for $U_{n}$.

Now assume that $U_{n} \subset C^{n}[a, b]$ is an extended Chebyshev system for $\{a, b\}$. The aim of this paper is to present sufficient conditions for the existence of a Bernstein operator $B_{n}: C[a, b] \rightarrow U_{n}$ generalizing the classical Bernstein operator

$$
\left(B_{n} f\right)(x)=\sum_{k=0}^{n} f\left(\frac{k}{n}\right)\left(\begin{array}{l}
n \\
k
\end{array}\right) x^{k}(1-x)^{n-k},
$$

in the case $U_{n}$ is the set of all polynomials of degree $\leq n$ on $[0,1]$. Here $B_{n} 1=1$ and $B_{n} x=x$. For a general $U_{n}$, we assume that a Bernstein basis $p_{n, k}, k=0, \ldots, n$, exists, and we consider linear operators $B_{n}: C[a, b] \rightarrow U_{n}$ of the form

$$
B_{n} f=\sum_{k=0}^{n} f\left(t_{k}\right) \alpha_{k} p_{n, k}
$$

with points $t_{0}, \ldots, t_{n} \in[a, b]$ and positive numbers $\alpha_{0}, \ldots, \alpha_{n}$. We determine the points $t_{0}, \ldots, t_{n}$ and the coefficients $\alpha_{0}, \ldots, \alpha_{n}$ from the property that $B_{n}$ fixes two given functions $f_{0}, f_{1} \in U_{n}$, i.e. from

$$
B_{n} f_{0}=f_{0} \text { and } B_{n} f_{1}=f_{1} \text {. }
$$

Throughout the paper we shall assume that $f_{0}$ is strictly positive (i.e., $f_{0}$ is real-valued and $\left.f_{0}>0\right)$ on $[a, b]$ and that $f_{1} / f_{0}$ is strictly increasing on $[a, b]$. Condition (2) leads to equations for the nodes $t_{0}, \ldots, t_{n}$ and the coefficients $\alpha_{0}, \ldots, \alpha_{n}$, cf. (91) and (10), which have at most one solution. The main difficulty is to guarantee the solvability of these equations, something that leads to additional assumptions.

In order to guarantee that the Bernstein operator defined in (1) is positive (i.e., for all non-negative $\left.f \in C[a, b], B_{n} f \geq 0\right)$ we shall assume that the Bernstein basis is nonnegative, that is, $p_{n, k}(x) \geq 0$ for all $x \in[a, b]$ and $k=0, \ldots, n$. Proposition 4 in [1] shows that an extended Chebyshev system $U_{n}$ for $\{a, b\}$ possesses a Bernstein basis of realvalued functions if and only if $U_{n}$ is closed under complex conjugation, i.e. the complex conjugate function $\bar{f}$ is in $U_{n}$ for all $f \in U_{n}$. If $U_{n}$ is an extended Chebyshev system over the interval $[a, b]$, closed under complex conjugation, then the Bernstein basis functions $p_{n, k}$ are real-valued and do not have zeros in the open interval $(a, b)$. Thus, under these assumptions, non-negative Bernstein basis exist.

In order to formulate our main result we need the following notation: For a strictly positive function $f_{0} \in U_{n} \subset C^{n}[a, b]$ we define the space of derivatives modulo $f_{0}$ by

$$
D_{f_{0}} U_{n}:=\left\{\frac{d}{d x}\left(\frac{f}{f_{0}}\right): f \in U_{n}\right\} .
$$


Clearly $D_{f_{0}} U_{n}$ is a linear space of dimension $n$. Next we state our main result. It is an immediate consequence of Theorems 2 , and 8 .

Theorem 1. Assume that $U_{n}$ possesses a non-negative Bernstein basis $p_{n, k}, k=0, \ldots, n$ for $\{a, b\} \subset I, a<b$. Let $f_{0} \in U_{n}$ be strictly positive, suppose $f_{1} \in U_{n}$ has the property that $f_{1} / f_{0}$ is strictly increasing, and assume that $D_{f_{0}} U_{n}$ possesses a non-negative Bernstein basis $q_{n-1, k}, k=0, \ldots, n-1$. If the coefficients $w_{k}, k=0, \ldots, n-1$, defined by

$$
\frac{d}{d x} \frac{f_{1}}{f_{0}}=\sum_{k=0}^{n-1} w_{k} q_{n-1, k}
$$

are non-negative, then there exist points $t_{0}, \ldots, t_{n} \in[a, b]$ with $t_{0}=a$ and $t_{n}=b$ and positive coefficients $\alpha_{0}, \ldots, \alpha_{n}$, such that the operator

$$
B_{n} f=\sum_{k=0}^{n} f\left(t_{k}\right) \alpha_{k} p_{n, k}
$$

satisfies $B_{n} f_{0}=f_{0}$ and $B_{n} f_{1}=f_{1}$.

The paper is organized as follows: In the second section we shall give a simple characterization of the existence of a Bernstein operator fixing two functions, in terms of properties of the Bernstein basis coefficients in the expansions of $f_{0}$ and $f_{1}$. Section 3 contains some basic results leading to an alternative proof of the normalization property established in [4] and [15. The main result in Section 4 is Theorem [8, from which Theorem 1 follows. Section 5 is devoted to the case where $U_{n}$ is the space of exponential polynomials; we present improvements of some previous results from [1]. In the last section we shall exhibit an example showing that the assumption $w_{k} \geq 0$ in (3) is necessary for Theorem 1 to hold.

\section{On the EXISTENCE OF Bernstein OPERATORS}

Let us review some basic facts and notations. The $k$-th derivative of a function $f$ is denoted by $f^{(k)}$. A function $f \in C^{n}(I, \mathbb{C})$ has a zero of order $k$ or of multiplicity $k$ at a point $a \in I$ if $f(a)=\ldots=f^{(k-1)}(a)=0$ and $f^{(k)}(a) \neq 0$. We shall repeatedly use the fact that

$$
k ! \cdot \lim _{x \rightarrow a} \frac{f(x)}{(x-a)^{k}}=f^{(k)}(a) .
$$

for any function $f \in C^{(k)}(I)$ with $f(a)=\ldots=f^{(k-1)}(a)=0$. Of course, the same formula holds for one side limits, which is the way we will use it.

If $p_{n, k}, k=0, \ldots, n$, is a Bernstein basis of $U_{n}$, then given any two functions $f_{0}, f_{1} \in U_{n}$ there exist coefficients $\beta_{0}, \ldots, \beta_{n}$ and $\gamma_{0}, \ldots, \gamma_{n}$ such that

$$
f_{0}(x)=\sum_{k=0}^{n} \beta_{k} p_{n, k}(x) \text { and } f_{1}(x)=\sum_{k=0}^{n} \gamma_{k} p_{n, k}(x) \text {. }
$$


Next we characterize the existence of a Bernstein operator fixing $f_{0}, f_{1} \in U_{n}$, in terms of properties of the coefficients $\beta_{0}, \ldots, \beta_{n}$ and $\gamma_{0}, \ldots, \gamma_{n}$ :

Theorem 2. Assume that a subspace $U_{n} \subset C^{n}[a, b]$ of dimension $n+1$ possesses a non-negative Bernstein basis $p_{n, k}, k=0, \ldots, n$, for $\{a, b\}$. Suppose $f_{0}, f_{1} \in U_{n}$ are such that $f_{0}>0$ and $f_{1} / f_{0}$ is strictly increasing on $[a, b]$. Then there exist (unique) points $t_{0}, \ldots, t_{n} \in[a, b]$ and positive coefficients $\alpha_{0}, \ldots, \alpha_{n}$ such that the operator

$$
B_{n} f=\sum_{k=0}^{n} f\left(t_{k}\right) \alpha_{k} p_{n, k}
$$

fixes $f_{0}$ and $f_{1}$, if and only if the coefficients defined in (6) satisfy $\beta_{k}>0$ and

$$
\frac{f_{1}(a)}{f_{0}(a)}=\frac{\gamma_{0}}{\beta_{0}} \leq \frac{\gamma_{k}}{\beta_{k}} \leq \frac{\gamma_{n}}{\beta_{n}}=\frac{f_{1}(b)}{f_{0}(b)}
$$

for all $k=0, \ldots, n$.

Proof. Suppose there exists a Bernstein operator $B_{n}$ with the properties described by the theorem. From $B_{n}\left(f_{0}\right)=f_{0}$ and (6) we get

$$
B_{n}\left(f_{0}\right)=\sum_{k=0}^{n} f_{0}\left(t_{k}\right) \alpha_{k} p_{n, k}=\sum_{k=0}^{n} \beta_{k} p_{n, k} .
$$

Since $p_{n, k}$ is a basis we conclude that

$$
f_{0}\left(t_{k}\right) \alpha_{k}=\beta_{k}
$$

A similar argument, using $B_{n} f_{1}=f_{1}$, yields $f_{1}\left(t_{k}\right) \alpha_{k}=\gamma_{k}$. Dividing by $f_{0}\left(t_{k}\right) \alpha_{k}=\beta_{k}$, we find that $t_{k}$ satisfies

$$
\frac{f_{1}\left(t_{k}\right)}{f_{0}\left(t_{k}\right)}=\frac{\gamma_{k}}{\beta_{k}}
$$

From $f_{0}>0$ and $\alpha_{k}>0$ we get $\beta_{k}>0$. Inserting $x=a$ in (6) we obtain $f_{0}(a)=\beta_{0} p_{n, 0}(a)$ and $f_{1}(a)=\gamma_{0} p_{n, 0}(a)$. Thus

$$
\frac{f_{1}(a)}{f_{0}(a)}=\frac{\gamma_{0}}{\beta_{0}}
$$

Similarly, $f_{0}(b)=\beta_{n} p_{n, n}(b)$ and $f_{1}(b)=\gamma_{n} p_{n, n}(b)$ imply that $\frac{f_{1}(b)}{f_{0}(b)}=\frac{\gamma_{n}}{\beta_{n}}$. By assumption, $f_{1} / f_{0}$ is increasing and $t_{k} \in[a, b]$, so $\frac{\gamma_{0}}{\beta_{0}} \leq \frac{\gamma_{k}}{\beta_{k}} \leq \frac{\gamma_{n}}{\beta_{n}}$ for all $k=0, \ldots, n$.

Next, suppose that the coefficients $\beta_{0}, \ldots, \beta_{n}$ are positive and (8) is satisfied. Since $h:=$ $f_{1} / f_{0}$ is strictly increasing and continuous, the image of $h$ is just the interval $[h(a), h(b)]$. So for each $\gamma_{k} / \beta_{k}$ there exists a unique point $t_{k} \in[a, b]$ such that $h\left(t_{k}\right)=\gamma_{k} / \beta_{k}, k=$ $0, \ldots, n$. We define $\alpha_{k}>0$ by the equation $f_{0}\left(t_{k}\right) \alpha_{k}=\beta_{k}$, using $f_{0}>0$. Then

$$
B_{n}\left(f_{0}\right)=\sum_{k=0}^{n} f_{0}\left(t_{k}\right) \alpha_{k} p_{n, k}=\sum_{k=0}^{n} \beta_{k} p_{n, k}=f_{0} .
$$


Finally, it follows from the equations $h\left(t_{k}\right)=\gamma_{k} / \beta_{k}$ and $f_{0}\left(t_{k}\right) \alpha_{k}=\beta_{k}$ that

$$
B_{n}\left(f_{1}\right)=\sum_{k=0}^{n} f_{1}\left(t_{k}\right) \alpha_{k} p_{n, k}=\sum_{k=0}^{n} f_{0}\left(t_{k}\right) \frac{\gamma_{k}}{\beta_{k}} \alpha_{k} p_{n, k}=\sum_{k=0}^{n} \gamma_{k} p_{n, k}=f_{1} .
$$

\section{THE NORMALIZATION PROPERTY}

A remarkable recent result is the existence of a so-called normalized Bernstein basis: Assume that $U_{n} \subset C^{n}(I, \mathbb{R})$ is an extended Chebyshev system over $[a, b]$ containing the constant function 1 , so there exists a non-negative Bernstein basis $p_{n, k}, k=0, \ldots, n$. In particular, for some coefficients $\alpha_{k}, k=0, \ldots, n$, we have $1=\sum_{k=0}^{n} \alpha_{k} p_{n, k}$. The normalization property says that the coefficients $\alpha_{k}$ are positive, (i.e.,, no cancellation is required in order to obtain the constant function 1 from the basis). It is proved in [4] and [15] that the normalization property holds if and only if the space $U_{n}^{\prime}$ of all derivatives $f^{\prime}$ with $f \in U_{n}$ is an extended Chebyshev space over $[a, b]$. As a byproduct of our investigations we shall obtain an alternative proof of this fact, valid in the more general context of subspaces of $C^{n}(I, K)$.

Proposition 3. Assume that $U_{n}$ has a Bernstein basis $p_{n, k}, k=0, \ldots, n$. Let $f_{0} \in U_{n}$ be strictly positive and suppose that $D_{f_{0}} U_{n}$ has a Bernstein basis $q_{n-1, k}, k=0, \ldots, n-1$. Set $c_{0}:=0, q_{n-1,-1}:=0, d_{n}:=0$, and $q_{n-1, n}:=0$. For $k=1, \ldots, n$, define the non-zero numbers

$$
c_{k}:=\frac{1}{f_{0}(a)} \lim _{x \downarrow a} \frac{\frac{d}{d x} p_{n, k}(x)}{q_{n-1, k-1}(x)}=\frac{1}{f_{0}(a)} \frac{p_{n, k}^{(k)}(a)}{q_{n-1, k-1}^{(k-1)}(a)}
$$

and for $k=0, \ldots, n-1$, the non-zero numbers

$$
d_{k}:=\frac{1}{f_{0}(b)} \lim _{x \uparrow b} \frac{\frac{d}{d x} p_{n, k}(x)}{q_{n-1, k}(x)}=\frac{1}{f_{0}(b)} \frac{p_{n, k}^{(n-k)}(b)}{q_{n-1, k}^{(n-1-k)}(b)} .
$$

Then for every $k=0, \ldots, n$,

$$
\frac{d}{d x} \frac{p_{n, k}(x)}{f_{0}(x)}=c_{k} q_{n-1, k-1}(x)+d_{k} q_{n-1, k}(x) .
$$

Proof. Since $\left(p_{n, k} / f_{0}\right)^{\prime} \in D_{f_{0}}\left(U_{n}\right)$, there exist scalars $w_{k, i}$ such that

$$
F_{k}(x):=\frac{d}{d x} \frac{p_{n, k}(x)}{f_{0}(x)}=\sum_{i=0}^{n-1} w_{k, i} q_{n-1, i}(x) .
$$


We claim that $F_{n}$ has a zero of order $n-1$ at $a$. Indeed, using (5) first with $f=p_{n, n} / f_{0}$ and then with $f=p_{n, n}$, we see that

$$
F_{n}^{(k-1)}(a)=\frac{d^{k}}{d x^{k}} \frac{p_{n, n}}{f_{0}}(a)=k ! \lim _{x \rightarrow a} \frac{p_{n, n}(x)}{(x-a)^{k} f_{0}(x)}=\frac{k !}{f_{0}(a)} \lim _{x \rightarrow a} \frac{p_{n, n}(x)}{(x-a)^{k}}=\frac{p_{n, n}^{(k)}(a)}{f_{0}(a)}
$$

Since $p_{n, n}^{(k)}(a)=0$ for $k=1, \ldots, n-1$ and $p_{n, n}^{(k)}(a) \neq 0$ when $k=n$, the claim follows. Renaming $c_{n}:=w_{n, n-1}$, we see that (13) holds when $k=n$, for $q_{n-1, k}$ has exactly $k$ zeros at $a$, so $F_{n}(x)=w_{n, n-1} q_{n-1, n-1}(x)$. Now the first equality in (111) (when $k=n$ ) follows immediately. Differentiating $n-1$ times both sides of $F_{n}(x)=c_{n} q_{n-1, n-1}(x)$ and using (15) we obtain the second equality in (11), for $k=n$.

The corresponding results when $k=0, \ldots, n-1$ are obtained in an entirely analogous way; in particular, for $k=1, \ldots, n-1$, since $F_{k}$ has a zero of order $k-1$ at $a$ and a zero of order $n-k-1$ at $b$ for $k=1, \ldots, n-1$, we conclude that $F_{k}=w_{k, k-1} q_{n-1, k-1}+w_{k, k} q_{n-1, k}$. Then we rename $c_{k}=w_{k, k-1}, d_{k}=w_{k, k}$, and argue as before.

Theorem 4. Assume that $U_{n}$ has a Bernstein basis $p_{n, k}, k=0, \ldots, n$. Let $f_{0} \in U_{n}$ be strictly positive and suppose $D_{f_{0}} U_{n}$ has a Bernstein basis $q_{n-1, k}, k=0, \ldots, n-1$. Let $c_{1}, \ldots, c_{n}$ and $d_{0}, \ldots, d_{n-1}$ be the non-zero numbers defined in (11) and (12). Then

$$
f_{0}(x)=\frac{f_{0}(a)}{p_{n, 0}(a)} p_{n, 0}(x)+\sum_{k=1}^{n}(-1)^{k} \frac{d_{0} \cdots d_{k-1}}{c_{1} \cdots c_{k}} \frac{f_{0}(a)}{p_{n, 0}(a)} p_{n, k}(x) .
$$

Proof. For some coefficients $\beta_{0}, \ldots, \beta_{n}$ we have $f_{0}(x)=\sum_{k=0}^{n} \beta_{k} p_{n, k}(x)$. Inserting $x=a$ yields $f_{0}(a)=\beta_{0} p_{n, 0}(a)$. Since

$$
1=\sum_{k=0}^{n} \beta_{k} \frac{p_{n, k}(x)}{f_{0}(x)}
$$

from Proposition 3 we obtain

$$
0=\frac{d}{d x} 1=\sum_{k=0}^{n-1}\left(\beta_{k+1} c_{k+1}+\beta_{k} d_{k}\right) q_{n-1, k}
$$

Thus, $\beta_{k+1}=-\beta_{k} d_{k} / c_{k+1}$ for $k=0, \ldots, n-1$, so $\beta_{k}=(-1)^{k} \beta_{0}\left(d_{0} \cdots d_{k-1}\right) /\left(c_{1} \cdots c_{k}\right)$ when $k=1, \ldots, n$, and (16) follows.

Next we want to discuss the normalization property, so we ask when the coefficients defined in (16) are positive.

Definition 5. Let $U_{n} \subset C^{n}[a, b]$ be an extended Chebyshev system for $\{a, b\}$. We say that a Bernstein basis $p_{n, k}$ for $\{a, b\}, k=0, \ldots, n$, is locally non-negative at $\{a, b\}$ if there exists a $\delta>0$ such that for all $k=0, \ldots, n$ and all $x \in[a, a+\delta) \cup(b-\delta, b]$ we have $p_{n, k}(x) \geq 0$. 
Let us give an example of a locally non-negative Bernstein basis for which non-negativity fails:

Example 1: Let $U_{2}$ be the linear space generated by the functions $1, \cos x, \sin x$. If $b \neq 2 \pi k, k \in \mathbb{N}$, then $U_{2}$ does possess a Bernstein basis for $\{0, b\}$, given by

$$
\begin{aligned}
& p_{2,2}(x)=1-\cos x \\
& p_{2,1}(x)=\sin x-\frac{\sin b}{1-\cos b}(1-\cos x), \\
& p_{2,0}(x)=1-\cos (x-b)=1-\cos b \cos x-\sin b \sin x .
\end{aligned}
$$

Furthermore, when $k \in \mathbb{N}, k \neq 0, U_{2}$ does not have a Bernstein basis for $\{0,2 \pi k\}$. We claim that $p_{2, k}, k=0,1,2$, is a locally non-negative Bernstein basis for $\{0, b\}$ whenever $0<b \neq 2 \pi k, k \in \mathbb{N}$. Indeed $p_{2,2}$ and $p_{2,0}$ are obviously non-negative. Since

$$
p_{2,1}^{\prime}(b)=\cos b-\frac{\sin b}{1-\cos b} \sin b=-1<0
$$

we see that $p_{2,1}$ is strictly decreasing in a neighborhood of $b$, so $p_{2,1}(x)>p_{2,1}(b)=0$ for $x<b$ sufficiently near to $b$. Similarly $p_{2,1}^{\prime}(0)>0$ implies that $p_{2,1}(x)>0$ for all $x>0$ sufficiently near to 0.

Finally, for suitably chosen values of $b$ the non-negativity of $p_{2,1}$ fails (but not for all values, consider, say, $b=\pi)$. Select, for instance, $b \approx 3 \pi$. Then $\sin b \approx 0$ and $1-\cos b \approx 2$, so $p_{2,1}$ is just $\sin x$ plus a small perturbation, and hence it changes sign over $[0, b]$.

Lemma 6. Let $p_{n, k}, k=0, \ldots, n$, be a locally non-negative Bernstein basis for $\{a, b\}$. Then there exists a $\delta>0$ such that $p_{n, k}^{\prime}(x)<0$ for all $x \in[b-\delta, b]$ and all $k=0, \ldots, n-1$, while $p_{n, k}^{\prime}(x)>0$ for all $x \in[a, a+\delta]$ and all $k=1, \ldots, n$. Thus, the numbers $c_{k}$ defined in (11) for $k=1, \ldots, n$ are positive, and the numbers $d_{k}$ defined in (12) for $k=0, \ldots, n-1$ are negative.

Proof. We prove the result about $p_{n, k}^{\prime}$ only for the right endpoint $b$, since the arguments for $a$ are entirely analogous. Let us write $p_{n, k}(x)=(b-x)^{n-k-1} g(x)$ for $k=0, \ldots, n-1$. Since $p_{n, k}$ has a zero of order $n-k$ at $b$ it is clear that $g(b)=0$. Furthermore $g(x) \geq 0$ on $[b-\tau, b]$ for some $\tau>0$, since $p_{n, k}$ has this property. Then for $x \in(b-\tau, b)$

$$
\frac{g(x)-g(b)}{x-b}=\frac{g(x)}{x-b} \leq 0 .
$$

Taking the limit as $x \rightarrow b$ we see that $g^{\prime}(b) \leq 0$. Since $g$ has only one zero at $b$ we conclude that $g^{\prime}(b)<0$. By continuity we infer that $g^{\prime}(x)<0$ on some interval $(b-\varepsilon, b)$. Hence

$$
p_{n, k}^{\prime}(x)=-(n-k-1)(b-x)^{n-k-2} g(x)+(b-x)^{n-k-1} g^{\prime}(x)
$$

and from this we see that $p_{n, k}^{\prime}(x)<0$ on $(b-\varepsilon, b)$. The preceding argument gives one $\varepsilon$ for each $k=0, \ldots, n-1$. To find $\delta$, select the smallest such $\varepsilon$. 
Next, recalling that $c_{k}=\lim _{x \downarrow a} p_{n, k}^{\prime}(x) /\left(f_{0}(a) q_{n-1, k-1}(x)\right)$, cf. (11), and that $d_{k}:=$ $\lim _{x \uparrow b} p_{n, k}^{\prime}(x) /\left(f_{0}(b) q_{n-1, k}(x)\right)$, cf. (12), we obtain $c_{k}>0$ and $d_{k}<0$.

Using Theorem 4 we give a simple proof, in a more general setting, of the normalization property established in [4] and [15]. Note that $f_{0}$ need not be constant, it is enough to assume $f_{0}>0$ to obtain the result. Considering functions more general than 1 will be useful later on, when dealing with exponential polynomials.

Theorem 7. Assume that $U_{n}$ possesses a locally non-negative Bernstein basis $p_{n, k}, k=$ $0, \ldots, n$ for $a \neq b$. Let $f_{0} \in U_{n}$ be strictly positive and assume that $D_{f_{0}} U_{n}$ possesses a locally non-negative Bernstein basis $q_{n-1, k}, k=0, \ldots, n-1$. Then the coefficients $\beta_{0}, \ldots, \beta_{n}$ in the expansion $f_{0}=\sum_{k=0}^{n} \beta_{k} p_{n, k}$ are positive.

Proof. By Theorem 4 it suffices to show that for $k=1, \ldots, n, c_{k}>0$ and for $k=$ $0, \ldots, n-1, d_{k}<0$. But this is the content of Lemma 6 .

\section{Proof of the MAIN RESUlt}

We now want to prove the main result of the paper, Theorem 1, presented in the introduction. The following theorem shows that condition (8) in Theorem 2 is satisfied, so Theorem 2 implies Theorem 1.

As in ([6), we use the notation

$$
f_{0}(x)=\sum_{k=0}^{n} \beta_{k} p_{n, k}(x) \text { and } f_{1}(x)=\sum_{k=0}^{n} \gamma_{k} p_{n, k}(x),
$$

for the functions $f_{0}, f_{1} \in U_{n}$ with the properties indicated in the next theorem.

Theorem 8. Assume that $U_{n}$ possesses a locally non-negative Bernstein basis $p_{n, k}, k=$ $0, \ldots, n$, for $\{a, b\}$. Suppose $f_{0}, f_{1} \in U_{n}$ are such that $f_{0}>0$ and $f_{1} / f_{0}$ is strictly increasing on $[a, b]$. If $D_{f_{0}} U_{n}$ has a locally non-negative Bernstein basis $q_{n-1, k}, k=0, \ldots, n-1$, then

$$
\frac{f_{1}(a)}{f_{0}(a)}=\frac{\gamma_{0}}{\beta_{0}} \leq \frac{\gamma_{k}}{\beta_{k}} \leq \frac{\gamma_{n}}{\beta_{n}}=\frac{f_{1}(b)}{f_{0}(b)}
$$

for $k=1$ and $k=n-1$. If the coefficients $w_{k}, k=0, \ldots, n-1$, defined by

$$
\frac{d}{d x} \frac{f_{1}}{f_{0}}=\sum_{k=0}^{n-1} w_{k} q_{n-1, k}
$$

are non-negative, then (17) holds for all $k=1, \ldots, n-1$. If $w_{k}$ is positive for $k=0, \ldots, n-1$ then strict inequalities hold in (17).

Proof. Since $p_{n, k}, k=0, \ldots, n$, is a basis, there exists numbers $\delta_{1}, \ldots, \delta_{n}$ such that

$$
\psi_{a}:=f_{1}-\frac{f_{1}(a)}{f_{0}(a)} f_{0}=\sum_{k=0}^{n} \delta_{k} p_{n, k} .
$$


From (마) we get $\delta_{k}=\gamma_{k}-\frac{f_{1}(a)}{f_{0}(a)} \beta_{k}$ for $k=0, \ldots, n$. Since $\psi_{a}(a)=0$, we see that $\delta_{0}=0$ and therefore $\frac{\gamma_{0}}{\beta_{0}}=\frac{f_{1}(a)}{f_{0}(a)}$. If we can show that $\delta_{k} \geq 0$ for $k=1, \ldots, n$, then we obtain $\frac{\gamma_{0}}{\beta_{0}} \leq \frac{\gamma_{k}}{\beta_{k}}$. Similarly, $\delta_{k}>0$ implies that $\frac{\gamma_{0}}{\beta_{0}}<\frac{\gamma_{k}}{\beta_{k}}$ for $k=1, \ldots, n$. Let us prove the non-negativity (positivity) of $\delta_{k}$ for $k=1, \ldots, n$ from the corresponding assumptions for $w_{k-1}$. By writing $\frac{f_{1}}{f_{0}}-\frac{f_{1}(a)}{f_{0}(a)}=\sum_{k=1}^{n} \delta_{k} \frac{p_{n, k}}{f_{0}}$ and taking the derivative we obtain

$$
\frac{d}{d x} \frac{f_{1}}{f_{0}}=\sum_{k=1}^{n} \delta_{k} \frac{d}{d x}\left(\frac{p_{n, k}}{f_{0}}\right)
$$

Proposition 3 shows that $\frac{d}{d x} \frac{f_{1}}{f_{0}}=\sum_{k=1}^{n-1} \delta_{k}\left[c_{k} q_{n-1, k-1}+d_{k} q_{n-1, k}\right]+c_{n} \delta_{n} q_{n-1, n-1}$. A simple calculation gives

$$
\frac{d}{d x} \frac{f_{1}}{f_{0}}=\delta_{1} c_{1} q_{n-1,0}+\sum_{k=1}^{n-1}\left(\delta_{k+1} c_{k+1}+\delta_{k} d_{k}\right) q_{n-1, k} .
$$

On the other hand, we have the representation (18). Comparing coefficients yields $\delta_{1} c_{1}=$ $w_{0}$ and

$$
c_{k+1} \delta_{k+1}=w_{k}-\delta_{k} d_{k}
$$

for $k=1, \ldots, n-1$. Inserting $x=a$ in (18), we get

$$
\frac{d}{d x} \frac{f_{1}}{f_{0}}(a)=w_{0} q_{n-1,0}(a) \text {. }
$$

Recall that by Lemma 6, $c_{k}>0$ for $k=1, \ldots, n$ and $d_{k}<0$ for $k=0, \ldots, n-1$. Now $f_{1} / f_{0}$ is increasing and $q_{n-1,0}(a)>0$, so $w_{0} \geq 0$, and therefore $\delta_{1} \geq 0$ (since $c_{1}$ is positive). If $w_{k} \geq 0$, it follows from (20), by induction over $k \geq 1$, that $\delta_{k+1} \geq 0$ (since $c_{k+1}>0$ and $\left.d_{k}<0\right)$. Likewise, $\delta_{k+1}>0$ when $w_{0}, \ldots, w_{n}$ are strictly positive.

The proof of inequality $\frac{\gamma_{k}}{\beta_{k}} \leq \frac{\gamma_{n}}{\beta_{n}}$ follows the same path. We write

$$
\psi_{b}:=f_{1}-\frac{f_{1}(b)}{f_{0}(b)} f_{0}=\sum_{k=0}^{n} \Delta_{k} p_{n, k},
$$

and note that it suffices to prove $\Delta_{k}<0$ for $k=0, \ldots, n$, since $\gamma_{k}-\frac{f_{1}(b)}{f_{0}(b)} \beta_{k}=\Delta_{k}$. From $\psi_{b}(b)=0$ we infer that $\Delta_{n}=0$, and therefore $\frac{f_{1}(b)}{f_{0}(b)}=\frac{\gamma_{n}}{\beta_{n}}$. As before, Proposition 3 shows that

$$
\frac{d}{d x} \frac{f_{1}}{f_{0}}=\Delta_{n-1} d_{n-1} q_{n-1, n-1}+\sum_{k=0}^{n-2}\left(\Delta_{k+1} c_{k+1}+\Delta_{k} d_{k}\right) q_{n-1, k}
$$


Comparison of coefficients with those of (18) gives $w_{n-1}=\Delta_{n-1} d_{n-1}$ and the downward defined recursion $w_{k}=\Delta_{k+1} c_{k+1}+\Delta_{k} d_{k}$ for $k=n-2, \ldots, 0$. Since

$$
\Delta_{n-1} d_{n-1} q_{n-1, n-1}(b)=\frac{d}{d x} \frac{f_{1}}{f_{0}}(b) \geq 0,
$$

we have $\Delta_{n-1} d_{n-1} \geq 0$, and therefore $\Delta_{n-1} \leq 0$. Now we infer inductively that $\Delta_{k}=$ $d_{k}^{-1}\left(w_{k}-\Delta_{k+1} c_{k+1}\right) \leq 0$. Finally, when $w_{0}, \ldots, w_{n}>0$, the conclusiion $\Delta_{k}<0$ for $k=0, \ldots, n-1$ is obtained in the same way.

\section{Applications to exponential polynomials}

Let us recall that the space of exponential polynomials for given complex numbers $\lambda_{0}, \ldots, \lambda_{n}$ is defined by

$$
E_{\left(\lambda_{0}, \ldots, \lambda_{n}\right)}:=\left\{f \in C^{\infty}(\mathbb{R}, \mathbb{C}):\left(\frac{d}{d x}-\lambda_{0}\right) \ldots\left(\frac{d}{d x}-\lambda_{n}\right) f=0\right\} .
$$

We showed in [1] that a space $E_{\left(\lambda_{0}, \ldots, \lambda_{n}\right)}$, closed under complex conjugation, is an extended Chebyshev system over the interval $[a, b]$ provided $b-a<\pi / M_{n}$, where

$$
M_{n}:=\max \left\{\left|\operatorname{Im} \lambda_{j}\right|: j=0, \ldots, n\right\} .
$$

Under this condition, the following result is proved in [1] for real numbers $\lambda_{0}<\lambda_{1}$ : There exist unique ordered points $a=t_{0}<t_{1}<\ldots<t_{n}=b$, and unique positive coefficients $\alpha_{0}, \ldots, \alpha_{n}$, such that the operator $B_{n}: C[a, b] \rightarrow E_{\left(\lambda_{0}, \ldots, \lambda_{n}\right)}$ defined by (11) satisfies

$$
B_{n}\left(e^{\lambda_{0} x}\right)=e^{\lambda_{0} x} \text { and } B_{n}\left(e^{\lambda_{1} x}\right)=e^{\lambda_{1} x} .
$$

Next we show that under weaker assumptions, a slightly weaker conclusion (omitting the condition of ordered and distinct points $t_{0}<t_{1}<\ldots<t_{n}$ ) can be obtained from Theorem 1.

Theorem 9. Let $\lambda_{0}<\lambda_{1}$ be real numbers and suppose that $E_{\left(\lambda_{2}, \ldots, \lambda_{n}\right)}$ is an extended Chebyshev space for $[a, b]$, closed under complex conjugation. Then there exist unique points $t_{0}, \ldots, t_{n} \in[a, b]$ and unique positive coefficients $\alpha_{0}, \ldots, \alpha_{n}$, such that the operator $B_{n}: C[a, b] \rightarrow E_{\left(\lambda_{0}, \ldots, \lambda_{n}\right)}$ defined by (11) fixes $e^{\lambda_{0} x}$ and $e^{\lambda_{1} x}$.

Proof. Note that $E_{\left(\lambda_{0}, \lambda_{1}, \ldots, \lambda_{n}\right)}$ and $E_{\left(\lambda_{1}, \ldots, \lambda_{n}\right)}$ are extended Chebyshev spaces over the interval $[a, b]$, whenever $\lambda_{0}$ and $\lambda_{1}$ are real and $E_{\left(\lambda_{2}, \ldots, \lambda_{n}\right)}$ is an extended Chebyshev space for $[a, b]$ closed under complex conjugation (cf. the proof of Theorem 9 in [1]). Thus $U_{n}:=E_{\left(\lambda_{0}, \lambda_{1}, \ldots, \lambda_{n}\right)}$ possesses a non-negative Bernstein basis. We want to apply Theorem 1. Let $f_{0}(x)=e^{\lambda_{0} x}$, let $f_{1}(x)=e^{\lambda_{1} x}$, and observe that $g_{0}(x):=f_{1}(x) / f_{0}(x)=e^{\left(\lambda_{1}-\lambda_{0}\right) x}$ is strictly increasing, since $\lambda_{0}<\lambda_{1}$. Now

$$
\frac{d}{d x} \frac{f}{f_{0}}(x)=\frac{d}{d x}\left(f(x) e^{-\lambda_{0} x}\right)=e^{-\lambda_{0} x}\left(\frac{d}{d x}-\lambda_{0}\right) f(x),
$$


SO

$$
D_{f_{0}} E_{\left(\lambda_{0}, \ldots, \lambda_{n}\right)}=e^{-\lambda_{0} x} E_{\left(\lambda_{1}, \ldots, \lambda_{n}\right)}=E_{\left(\lambda_{1}-\lambda_{0}, \ldots, \lambda_{n}-\lambda_{0}\right)} .
$$

Applying this formula to $E_{\left(\lambda_{1}-\lambda_{0}, \ldots, \lambda_{n}-\lambda_{0}\right)}$ and $g_{0}(x)=e^{\left(\lambda_{1}-\lambda_{0}\right) x}$ we get

$$
D_{g_{0}} E_{\left(\lambda_{1}-\lambda_{0}, \ldots, \lambda_{n}-\lambda_{0}\right)}=E_{\left(\lambda_{2}-\lambda_{1}, \ldots, \lambda_{n}-\lambda_{1}\right)}=e^{-\lambda_{1} x} E_{\left(\lambda_{2}, \ldots, \lambda_{n}\right)} .
$$

It follows that $D_{f_{0}} E_{\left(\lambda_{0}, \ldots, \lambda_{n}\right)}$ is an extended Chebyshev system over $[a, b]$, so it has a nonnegative Bernstein basis $q_{n-1, k}, k=0, \ldots, n-1$. Finally, consider the coefficients $w_{k}$ in the representation

$$
g_{0}(x)=e^{\left(\lambda_{1}-\lambda_{0}\right) x}=\sum_{k=0}^{n-1} w_{k} q_{n-1, k}(x)
$$

Put $V_{n-1}:=D_{f_{0}} E_{\left(\lambda_{0}, \ldots, \lambda_{n}\right)}$ and apply Theorem 7 , By (23) $D_{g_{0}} V_{n-1}$ is an extended Chebyshev system over $[a, b]$, so by Theorem 7 the coefficients $w_{k}$ in (24) are positive. Since $g_{0}^{\prime}=\left(\lambda_{1}-\lambda_{0}\right) g_{0}$, we see that the coefficients defined by (3) are positive. Thus, Theorem 1 applies and the result follows.

Theorem 10. Let $\lambda_{0}$ be a real number and let $\lambda_{1}=\lambda_{0}$. Suppose that $E_{\left(\lambda_{2}, \ldots, \lambda_{n}\right)}$ is an extended Chebyshev space for $[a, b]$, closed under complex conjugation. Then there exist unique points $t_{0}, \ldots, t_{n} \in[a, b]$, and unique positive coefficients $\alpha_{0}, \ldots, \alpha_{n}$, such that the operator $B_{n}: C[a, b] \rightarrow E_{\left(\lambda_{0}, \lambda_{0}, \lambda_{2} \ldots, \lambda_{n}\right)}$ defined by (1) satisfies $B_{n} e^{\lambda_{0} x}=e^{\lambda_{0} x}$ and $B_{n}\left(x e^{\lambda_{0} x}\right)=x e^{\lambda_{0} x}$.

Proof. Set $f_{0}(x)=e^{\lambda_{0} x}$ and $f_{1}(x)=x e^{\lambda_{0} x}$. Then argue as in the preceding proof.

It is a natural question whether one can extend Theorem 9 to the case of two complex exponentials $e^{\lambda_{0} x}$ and $e^{\lambda_{1} x}$. Analyzing the first part of the proof of Theorem 2 (cf. (10) $)$ one obtains the following necessary condition for the node $t_{k}$

$$
e^{\left(\lambda_{1}-\lambda_{0}\right) t_{k}}=\frac{\gamma_{k}}{\beta_{k}}
$$

where $\beta_{k}$ and $\gamma_{k}$ are defined by the equations (6) for $k=0, \ldots, n$. Since $\beta_{k}, \gamma_{k}$ and $\lambda_{0}, \lambda_{1}$ are complex numbers there seems to be a priori little hope to find the possible solution $t_{k}$ in the interval $[a, b]$. However, in [18] S. Morigi and M. Neamtu are able to introduce a Bernstein operator, based on exponential polynomials with equidistant exponents, which fixes two complex exponentials $e^{\lambda_{0} x}$ and $e^{\lambda_{1} x}$ with $\lambda_{0}=\overline{\lambda_{1}}$. We generalize their result to the case of not necessarily equidistant exponents $\lambda_{0}, \ldots, \lambda_{n}$. Observe that in order for $e^{i x}, e^{-i x}$ to be an extended Chebyshev system over $[a, b]$, it is necessary to have $b-a<\pi$, as can be seen by counting the zeros of $\sin x$ (or $\cos x)$ in $[a, b]$. In general the corresponding condition on the length of $[a, b]$ is also sufficient, cf. Theorem 9 of [1].

Theorem 11. Let $\lambda_{0}, \ldots, \lambda_{n}$ be complex numbers such that $\lambda_{0}$ is not real and $\lambda_{1}=\overline{\lambda_{0}}$. Assume that the spaces $E_{\left(\lambda_{0}, \ldots, \lambda_{n}\right)}, E_{\left(\lambda_{2}, \ldots, \lambda_{n}\right)}$ and $E_{\left(\lambda_{0}, \lambda_{1}\right)}$ are extended Chebyshev systems over $[a, b]$, closed under complex conjugation. Then there exist unique points $t_{0}, \ldots, t_{n} \in$ 
$[a, b]$, and unique positive coefficients $\alpha_{0}, \ldots, \alpha_{n}$, such that the operator $B_{n}: C[a, b] \rightarrow$ $E_{\left(\lambda_{0}, \lambda_{1}, \lambda_{2} \ldots, \lambda_{n}\right)}$ defined by (11) satisfies $B_{n} e^{\lambda_{0} x}=e^{\lambda_{0} x}$ and $B_{n}\left(e^{\lambda_{1} x}\right)=e^{\lambda_{1} x}$.

Proof. Using [1], specifically Lemma 7 and Proposition 10 (which basically say that applying a translation to $\lambda_{0}, \ldots, \lambda_{n}$ preserves the extended Chebyshev system property, and the new Bernstein basis is obtained from the old one via multiplication by a suitable exponential function), one may assume that $\lambda_{0}$ is purely imaginary. Using the assumption that $E_{\left(\lambda_{0}, \lambda_{1}\right)}$ is an extended Chebyshev space we may assume that $\lambda_{0}=i, \lambda_{1}=-i$, and $[a, b] \subset\left(-\frac{1}{2} \pi, \frac{1}{2} \pi\right)$, by a scaling argument (cf. [1], Lemma 8, which states that multiplying each $\lambda_{0}, \ldots, \lambda_{n}$ by a fixed $c>0$ preserves, over a suitably re-scaled interval, the extended Chebyshev system property) and a translation of $[a, b]$ using a real number (cf. [1], Lemma 6 , by which such translation of $[a, b]$ preserves the extended Chebyshev system property).

Clearly $B_{n}$ fixes the functions $e^{i x}$ and $e^{-i x}$ if (and only if) $B_{n}$ fixes $f_{0}=\cos x$ and $f_{1}=\sin x$. Furthermore, $U_{n}:=\left\langle e^{i x}, e^{-i x}, e^{\lambda_{2} x}, \ldots, e^{\lambda_{n} x}\right\rangle=\left\langle f_{0}, f_{1}, e^{\lambda_{2} x}, \ldots, e^{\lambda_{n} x}\right\rangle$. Now we want to apply Theorem 1. By assumption $U_{n}=E_{\left(\lambda_{0}, \ldots, \lambda_{n}\right)}$ is an extended Chebyshev space over $[a, b]$, so it has a non-negative Bernstein basis. Next we consider the space $D_{f_{0}} U_{n}$. For every $h \in U_{n}$ we have

$$
\frac{d}{d x} \frac{h}{f_{0}}=\frac{h^{\prime} \cdot f_{0}-h \cdot f_{0}^{\prime}}{f_{0}^{2}} .
$$

Using $f_{0}^{\prime \prime}=-f_{0}$ we obtain

$$
\frac{d}{d x}\left(f_{0}^{2} \frac{d}{d x} \frac{h}{f_{0}}\right)=h^{\prime \prime} \cdot f_{0}-h \cdot f_{0}^{\prime \prime}=f_{0}\left(\frac{d^{2}}{d x^{2}}+1\right) h .
$$

Suppose now that $g \in D_{f_{0}} U_{n}$ had at least $n$ zeros in $[a, b]$. The function $g$ is of the form

$$
g=\frac{d}{d x} \frac{f}{f_{0}}
$$

for some $f \in U_{n}$, and $f_{0}^{2} g$ has at least $n$ zeros in $[a, b]$, so $\left(f_{0}^{2} g\right)^{\prime}$ has at least $n-1$ zeros in $[a, b]$ by Rolle's theorem. Note that $f \in U_{n}$ implies that

$$
\left(\frac{d^{2}}{d x^{2}}+1\right) f \in E_{\left(\lambda_{2}, \ldots, \lambda_{n}\right)}
$$

Thus by (26), with $h=f$, we have

$$
\left(f_{0}^{2} g\right)^{\prime} \in f_{0} \cdot E_{\left(\lambda_{2}, \ldots, \lambda_{n}\right)} .
$$

Since $E_{\left(\lambda_{2}, \ldots, \lambda_{n}\right)}$ is an extended Chebyshev space over $[a, b]$ we conclude that $\left(f_{0}^{2} g\right)^{\prime}=0$, so $f_{0}^{2} g$ is constant. But a constant with at least $n$ zeros is identically zero, so $g \equiv 0$, and we have shown that $D_{f_{0}} U_{n}$ is an extended Chebyshev space over $[a, b]$. Hence it has a 
non-negative Bernstein basis $q_{n-1, k}, k=0, \ldots, n-1$. Let us prove now the non-negativity of the coefficients $w_{k}$ in (3). Consider

$$
\frac{d}{d x} \frac{f_{1}}{f_{0}}=\frac{f_{1}^{\prime} \cdot f_{0}-f_{1} \cdot f_{0}^{\prime}}{f_{0}^{2}}=\frac{1}{f_{0}^{2}}=\sum_{k=0}^{n-1} w_{k} q_{n-1, k} .
$$

We show that the coefficients in the representation

$$
1=\sum_{k=0}^{n-1} w_{k} f_{0}^{2} \cdot q_{n-1, k}
$$

are positive. Since $V_{n-1}:=f_{0}^{2} \cdot D_{f_{0}} U_{n}$ obviously has the non-negative Bernstein basis $f_{0}^{2} \cdot q_{n-1, k}, k=0, \ldots, n-1$, and $g_{0}:=1$ is strictly positive, Theorem 7 tells us that the coefficients $w_{k}$ are positive provided that $D_{g_{0}} V_{n-1}$ is an extended Chebyshev space over $[a, b]$. Using (26) we obtain

$$
\begin{aligned}
D_{g_{0}} V_{n-1} & =\left\{\frac{d}{d x} h: h \in V_{n-1}\right\}=\left\{f_{0}\left(\frac{d^{2}}{d x^{2}}+1\right) f: f \in E_{\left(i,-i, \lambda_{2}, \ldots, \lambda_{n}\right)}\right\} \\
& =f_{0} \cdot E_{\left(\lambda_{2}, \ldots, \lambda_{n}\right)} .
\end{aligned}
$$

Since $E_{\left(\lambda_{2}, \ldots, \lambda_{n}\right)}$ is an extended Chebyshev sytem over $[a, b]$, so is $D_{g_{0}} V_{n-1}$, and the proof is complete.

\section{A counterexample}

The following counterexample shows that in order for Theorem 1 to hold, it is not enough to assume that $f_{0}>0$, that $f_{1} / f_{0}$ is increasing, and that both $U_{n}$ and $D_{f_{0}} U_{n}$ have non-negative Bernstein bases. Positivity of the coefficients $w_{k}$ in (3) is essential. Letting $f_{0} \equiv 1$, we shall exhibit a strictly increasing function $f_{1}$ on $[0, b] \subset[0,2 \pi)$ such that for all $b \geq 7 \pi / 4$, the node $t_{n-2}$ used in the definition of the Bernstein operator fixing $f_{0}$ and $f_{1}$, must fall outside $[0, b]$.

In the literature special attention has been devoted to the linear space generated by the functions $1, x, \ldots, x^{n-1}, \cos x, \sin x$ over the interval $[0, b], b>0$. It is well known that the linear space $\langle\cos x, \sin x\rangle$ is an extended Chebyshev space over $[0, b]$ whenever $b<\pi$, while $\langle 1, \cos x, \sin x\rangle$ and $\langle 1, x, \cos x, \sin x\rangle$ are extended Chebyshev spaces over $[0, b]$ if $b<2 \pi$. In [5] it is shown that

$$
U_{4}:=\left\langle 1, x, x^{2}, \cos x, \sin x\right\rangle
$$

is an extended Chebyshev system over $[0, b]$ provided that $b<\rho \approx 8.9868189$, where $\rho$ is the first positive zero of the equation $\tan (x / 2)=x / 2$.

We are going to present an explicit Bernstein basis $p_{4, k}, k=0, \ldots, 4$ for this specific space $U_{4}$ and $0<b<2 \pi$. Such a basis can be found, for instance, by using the algorithm 
from Section 3 of [1]. The first basis function

$$
p_{4,4}(x)=\cos x-1+\frac{1}{2} x^{2} \approx \frac{x^{4}}{24}
$$

is strictly positive for all real $x \neq 0$ and has a zero of order 4 at 0 . A basis function $p_{4,3}$ with three zeros at 0 and (at least) one zero at $b$ is given by

$$
p_{4,3}(x)=(\sin b-b)\left(\cos x-1+\frac{1}{2} x^{2}\right)+\left(\cos b-1+\frac{1}{2} b^{2}\right)(x-\sin x) .
$$

By either recalling that $U_{4}$ is an extended Chebyshev system over $[0, b]$, or directly by checking that if $b<\rho$, then $p_{4,3}^{\prime}(b) \neq 0$, we conclude that $p_{4,3}$ has exactly one zero at $b$. Furthermore, since $p_{4,3}$ does not change sign in $(0, b)$, to check positivity it is enough to evaluate $p_{4,3}$ at some suitably selected point. For instance, given $b \geq 7 \pi / 4$, it is clear that $p_{4,3}(\pi)>0$.

The basis function $p_{4,2}$ is given by

$$
\begin{aligned}
p_{4,2}(x)= & 1+\frac{2 \cos b-2+b \sin b}{b+b \cos b-2 \sin b} x-\frac{1}{2 b}\left(\sin b+\frac{2 \cos b-2+b \sin b}{b+b \cos b-2 \sin b}(1-\cos b)\right) x^{2} \\
& -\cos x-\frac{2 \cos b-2+b \sin b}{b+b \cos b-2 \sin b} \sin x .
\end{aligned}
$$

It is easy to see that $p_{4,2}$ has at least two zeros at 0 , and by computing $p_{4,2}(b)$ and $p_{4,2}^{\prime}(b)$, that it has at least two zeros at $b$. Hence, it has exactly two zeros at each endpoint of $[0, b]$, and none inside, so positivity follows by evaluating $p_{4,2}$ at some suitably chosen point in $(0, b)$ (alternatively, we mention that if $p_{4,2}$ were not positive we could replace it with $-p_{4,2}$ and nothing in the argument below would change). Finally, the basis functions $p_{4,0}$ and $p_{4,1}$ are defined by $p_{4,0}(x):=p_{4,4}(b-x)$ and $p_{4,1}(x):=p_{4,3}(b-x)$.

Let $f_{0}=1$, so

$$
D_{f_{0}} U_{4}=\langle 1, x, \cos x, \sin x\rangle .
$$

Thus, $U_{4}$ and $D_{f_{0}} U_{4}$ possess non-negative Bernstein bases for every $b \in(0,2 \pi)$. Next, consider the strictly increasing function $f_{1} \in U_{4}$ given by

$$
f_{1}(x):=1+x-\cos x .
$$

We want to show that for certain values $b \in(0,2 \pi)$ there does not exists a Bernstein operator (as described in Theorem 1) fixing $f_{0}$ and $f_{1}$. In order to facilitate the necessary computations we will start with some general remarks: Assume that $p_{n, k}, k=0, \ldots, n$, is a Bernstein basis of $U_{n}$ for $\{a, b\}$; let us compute some of the coefficients $\beta_{0}, \ldots, \beta_{n}$ in the expression

$$
f=\sum_{k=0}^{n} \beta_{k} p_{n, k}
$$


Inserting $x=b$ yields $p_{n, n}(b) \beta_{n}=f(b)$. Taking the derivative of $f$ at $b$ we get $f^{\prime}(b)=$ $\beta_{n-1} p_{n, n-1}^{\prime}(b)+\beta_{n} p_{n, n}^{\prime}(b)$, and after multiplying by $p_{n, n}(b)$, we obtain

$$
p_{n, n}(b) p_{n, n-1}^{\prime}(b) \beta_{n-1}=f^{\prime}(b) p_{n, n}(b)-f(b) p_{n, n}^{\prime}(b) \text {. }
$$

Consider the expression $f^{\prime \prime}(b)=\beta_{n-2} p_{n, n-2}^{\prime \prime}(b)+\beta_{n-1} p_{n, n-1}^{\prime \prime}(b)+\beta_{n} p_{n, n}^{\prime \prime}(b)$. Multiplication by $p_{n, n}(b) p_{n, n-1}^{\prime}(b)$ and a short computation shows that

$(27) \beta_{n-2} p_{n, n}(b) p_{n, n-1}^{\prime}(b) p_{n, n-2}^{\prime \prime}(b)=p_{n, n}(b)\left[f^{\prime \prime}(b) p_{n, n-1}^{\prime}(b)-f^{\prime}(b) p_{n, n-1}^{\prime \prime}(b)\right]+$

$$
f(b)\left[p_{n, n}^{\prime}(b) p_{n, n-1}^{\prime \prime}(b)-p_{n, n-1}^{\prime}(b) p_{n, n}^{\prime \prime}(b)\right] \text {. }
$$

Proposition 12. Let $n \geq 2$. Assume that $p_{n, k}, k=0, \ldots, n$ is a locally non-negative Bernstein basis of $U_{n}$ at $\{a, b\}$, and let $f_{0}, f_{1} \in U_{n}$ be given by $f_{0}=\sum_{k=0}^{n} \beta_{k} p_{n, k}$ and $f_{1}=\sum_{k=0}^{n} \gamma_{k} p_{n, k}$. If $f_{0}(b)>0$ and $\beta_{k}>0$ for $k=n, n-1, n-2$, then the requirement

$$
\frac{\gamma_{n-2}}{\beta_{n-2}} \leq \frac{\gamma_{n}}{\beta_{n}}
$$

is equivalent to the inequality

$$
\left[f_{0}(b) f_{1}^{\prime \prime}(b)-f_{0}^{\prime \prime}(b) f_{1}(b)\right] p_{n, n-1}^{\prime}(b)-\left[f_{0}(b) f_{1}^{\prime}(b)-f_{0}^{\prime}(b) f_{1}(b)\right] p_{n, n-1}^{\prime \prime}(b) \geq 0 .
$$

Proof. Since $p_{n, k}, k=0, \ldots, n$ is a locally non-negative Bernstein basis at $\{a, b\}$, it follows from Lemma 6 that $p_{n, n-1}^{\prime}(b)<0$, and $p_{n, n-2}^{\prime \prime}(b)>0$. Replacing $f$ in (27) by $f_{0}$, we get $B_{n-2}:=\beta_{n-2} p_{n, n}(b) p_{n, n-1}^{\prime}(b) p_{n, n-2}^{\prime \prime}(b)<0$, so we conclude that the condition

$$
\frac{G_{n-2}}{B_{n-2}}=\frac{\gamma_{n-2}}{\beta_{n-2}} \leq \frac{\gamma_{n}}{\beta_{n}}=\frac{f_{1}(b)}{f_{0}(b)},
$$

where $G_{n-2}:=\gamma_{n-2} p_{n, n}(b) p_{n, n-1}^{\prime}(b) p_{n, n-2}^{\prime \prime}(b)$, is equivalent to

$$
f_{0}(b) G_{n-2}-f_{1}(b) B_{n-2} \geq 0 \text {. }
$$

But this is just (29), as can be seen by setting $f=f_{0}$ and $f=f_{1}$ in (27).

Let us return to our example, where $f_{0}=1, f_{1}=1+x-\cos x$, and $n=4$. Fix $b \in[7 \pi / 4,2 \pi)$, and note that $\cos b>0, \sin b<0$, and $\cos b+\sin b \geq 0$. Now condition (29) becomes

$$
\begin{gathered}
h(b):=f_{1}^{\prime \prime}(b) p_{4,3}^{\prime}(b)-f_{1}^{\prime}(b) p_{4,3}^{\prime \prime}(b) \\
=(\cos b)\left(-(\sin b-b)^{2}+\left(\cos b-1+\frac{1}{2} b^{2}\right)(1-\cos b)\right) \\
-(1+\sin b)\left((\sin b-b)(1-\cos b)+\left(\cos b-1+\frac{1}{2} b^{2}\right)(\sin b)\right) \\
=(\cos b \sin b) b-\frac{1}{2} b^{2} \cos b+2 \cos ^{2} b-2 \cos b+b-b \cos b-\frac{1}{2}(\sin b) b^{2}+(\sin b) b-\frac{1}{2} b^{2} \\
<b-\frac{b^{2}}{2}<0 .
\end{gathered}
$$


Thus $\gamma_{2} / \beta_{2}>\gamma_{4} / \beta_{4}=f_{1}(b)$, and recalling (10), we see that the corresponding node $t_{2}=f_{1}^{-1}\left(\gamma_{2} / \beta_{2}\right)>b$, so it falls outside $[0, b]$.

\section{REFERENCES}

[1] J. M. Aldaz, O. Kounchev, H. Render, Bernstein operators for exponential polynomials, to appear in Constructive Approximation.

[2] J. M. Aldaz, O. Kounchev, H. Render, Shape preserving properties of Bernstein operators in Chebshev systems, in preparation.

[3] J. M. Carnicer, J.M. Peña, Shape preserving representations and optimality of the Bernstein basis, Adv. Comput. Math. 1 (1993), 173-196.

[4] J. M. Carnicer, E. Mainar, J.M. Peña, Critical Length for Design Purposes and Extended Chebyshev Spaces, Constr. Approx. 20 (2004), 55-71.

[5] J.M. Carnicer, E. Mainar, J.M. Peña, Shape preservation regions for six-dimensional space, Advances in Computational Mathematics 26 (2007), 121-136.

[6] P. Costantini, Curve and surface construction using variable degree polynomial splines, Comput. Aided Geom. Design 17 (2000), 419-446,

[7] P. Costantini, T. Lyche, C. Manni, On a class of weak Tchebycheff systems, Numer. Math. 101 (2005), 333-354.

[8] R.T. Farouki, T.N.T. Goodman, On the optimal stability of the Bernstein basis, Math. Comp. 65 (1996), 1553-1566.

[9] T.N.T. Goodman, M.L. Mazure, Blossoming beyond Chebyshev spaces, J. Approx. Theory 109 (2001), 48-81.

[10] D. Gonsor, M. Neamtu, Null spaces of Differential Operators, Polar Forms and Splines, J. Approx. Theory 86 (1996), 81-107.

[11] O. Kounchev, H. Render, New methods in Geometric Modelling and Controlling Exponential Processes, Proceedings of the Nato Advanced Research Workshop: Scientific Support for the Decision Making in the Security Sector, Velingrad, Bulgaria, 21-25.10.2006, (2007), 144-179.

[12] O. Kounchev, H. Render, On the Bernstein operator of S. Morigi and M. Neamtu, submitted.

[13] E. Mainar, J.M. Peña, J. Sánchez-Reyes, Shape perserving alternatives to the rational Bézier model, Comput. Aided Geom. Design 14 (1997), 5-11,

[14] M. Mazure, Bernstein bases in Müntz spaces, Numerical Algorithms 22 (1999), 285-304.

[15] M. Mazure, Chebyshev Spaces and Bernstein bases, Constr. Approx. 22 (2005), 347-363.

[16] M. Mazure, On the Hermite Interpolation, C.R. Acad. Sci. Paris, Ser. I, 340 (2005), 177-180.

[17] M. Mazure, H. Pottmann, Tchebycheff curves. Total positivity and its applications (Jaca, 1994), 187-218, Math. Appl., 359, Kluwer Acad. Publ., Dordrecht, 1996.

[18] S. Morigi, M. Neamtu, Some results for a class of generalized polynomials, Adv. Comput. Math. 12 (2000), 133-149.

[19] J.M. Peña, On the optimal stability of bases of univariate functions, Numer. Math. 91 (2002), 305318.

[20] J.M. Peña, On Descartes' rules of signs and their exactness, Math. Nachr. 278 (2005), 1706-1713.

[21] J. Zhang, C-curves: an extension of cubic curves, Comput. Aided Geom. Design 13 (1996), 199-217.

J. M. Aldaz: Permanent address: Departamento de Matemáticas y Computación, Universidad de La Rioja, 26004 Logroño, La Rioja, Spain.

E-mail address: jesus.munarrizaldaz@dmc.unirioja.es 
CURRENT ADDRESS: Departamento de Matemáticas, Universidad Autónoma de Madrid, Cantoblanco 28049, Madrid, Spain.

E-mail address: jesus.munarriz@uam.es

O. Kounchev: Institute of Mathematics and Informatics, Bulgarian Academy of Sciences, 8 Acad. G. Bonchev Str., 1113 Sofia, Bulgaria.

E-mail address: kounchev@gmx.de

H. Render: Departamento de Matemáticas y Computación, Universidad de la Rioja, Edificio Vives, Luis de Ulloa S/N., 26004 Logroño, España.

E-mail address: render@gmx.de 